University of Wollongong

Research Online

Australian Institute for Innovative Materials -

Papers

Australian Institute for Innovative Materials

$1-1-2008$

Optical property and electronic band structure of a piezoelectric compound Ga3P07 studied by the first-principles calculation

Zhenxiang Cheng

University of Wollongong, cheng@uow.edu.au

Xiaolin Wang

University of Wollongong, xiaolin@uow.edu.au

Follow this and additional works at: https://ro.uow.edu.au/aiimpapers

Part of the Engineering Commons, and the Physical Sciences and Mathematics Commons

Research Online is the open access institutional repository for the University of Wollongong. For further information contact the UOW Library: research-pubs@uow.edu.au 


\title{
Optical property and electronic band structure of a piezoelectric compound Ga3P07 studied by the first-principles calculation
}

\author{
Abstract \\ The structure, electronic and optical properties of a piezoelectric material, $\mathrm{Ga}_{3} \mathrm{PO}_{7}$ were studied by first- \\ principles calculations in the framework of density functional theory. The calculated structure is in \\ agreement with the experimental data. Band structure reveals that $\mathrm{Ga}_{3} \mathrm{PO}_{7}$ has a band gap of $3.69 \mathrm{eV}$. \\ Analysis of partial density of states and Mulliken charge population indicates existence of $\mathrm{GaO}_{5}$ and $\mathrm{PO}_{4}$ \\ anion groups in $\mathrm{Ga}_{3} \mathrm{PO}_{7}$. Furthermore, its optical properties, including dielectric constant, absorption, \\ reflectivity, refractive index, and electron loss were calculated and analysed, which show that $\mathrm{Ga}_{3} \mathrm{PO}_{7}$ has \\ potential applications based on combination of its piezoelectric and optical properties.

\section{Keywords} \\ Optical, property, electronic, band, structure, piezoelectric, compound, Ga3P07, studied, first, principles, \\ calculation \\ Disciplines \\ Engineering | Physical Sciences and Mathematics

\section{Publication Details} \\ Cheng, Z \& Wang, X (2008), Optical property and electronic band structure of a piezoelectric compound \\ Ga3P07 studied by the first-principles calculation, Applied Physics Letters, 92(26), pp. \\ 261915-1-261915-3.
}

This journal article is available at Research Online: https://ro.uow.edu.au/aiimpapers/109 


\title{
Optical property and electronic band structure of a piezoelectric compound $\mathrm{Ga}_{3} \mathrm{PO}_{7}$ studied by the first-principles calculation
}

\author{
Z. X. Cheng and X. L. Wang ${ }^{\text {a) }}$ \\ Spintronic and Electronic Materials Group, Institute for Superconducting and Electronic Materials, \\ University of Wollongong, New South Wales 2522, Australia
}

(Received 15 May 2008; accepted 17 June 2008; published online 3 July 2008)

\begin{abstract}
The structure, electronic, and optical properties of a piezoelectric material, $\mathrm{Ga}_{3} \mathrm{PO}_{7}$, were studied by first-principles calculations in the framework of density functional theory. The calculated structure is in agreement with the experimental data. Band structure reveals that $\mathrm{Ga}_{3} \mathrm{PO}_{7}$ has a band gap of $3.69 \mathrm{eV}$. Analysis of partial density of states and Mulliken charge population indicates existence of $\mathrm{GaO}_{5}$ and $\mathrm{PO}_{4}$ anion groups in $\mathrm{Ga}_{3} \mathrm{PO}_{7}$. Furthermore, its optical properties, including dielectric constant, absorption, reflectivity, refractive index, and electron loss were calculated and analyzed, which show that $\mathrm{Ga}_{3} \mathrm{PO}_{7}$ has potential applications based on combination of its piezoelectric and optical properties. (C) 2008 American Institute of Physics. [DOI: 10.1063/1.2955827]
\end{abstract}

$\mathrm{Ga}_{3} \mathrm{PO}_{7}$, having a noncentrosymmetric trigonal structure with the space group of $R 3 m$, provides great potential in applications based on its optical, piezoelectric, and pyroelectric properties. ${ }^{1}$ However, for a quite long time after $\mathrm{Ga}_{3} \mathrm{PO}_{7}$ was initially discovered, there were no further reports on its physical properties. This is mainly due to the difficulty in growing single crystals with sufficient size for measurement and application, especially optical measurement and application. Very recently, $\mathrm{Ga}_{3} \mathrm{PO}_{7}$ single crystals with sizes up to several centimeters have been grown by the high temperature flux method, which paves the way for further exploration of its optical and electro-optical properties, and eventually for practical applications. ${ }^{2}$ Therefore, it is practically meaningful to study its optical and electronic structures. In this work, we present a series of first-principles density function calculations on the structural, electronic, and optical properties of $\mathrm{Ga}_{3} \mathrm{PO}_{7}$ material.

The calculations were performed using the plane-wave pseudopotential method based on density functional theory (DFT) with the generalized gradient approximation (GGA) in the scheme of Perdew-Burke-Ernzerhof. ${ }^{3}$ The ionelectron interaction was modeled by ultrasoft Vanderbilt-type pseudopotentials. ${ }^{4}$ Mulliken charges were calculated according to the formalism described by Segall et al. ${ }^{5}$ A plane-wave cutoff energy of $400 \mathrm{eV}$ was employed throughout the calculation. For the sampling of the Brillouin zone, the electronic structures and optical properties use $5 \times 5 \times 5$ and 8 $\times 8 \times 8 k$-point grids, respectively, generated according to the Monkhorst-Pack scheme. ${ }^{6}$

The optical properties of $\mathrm{Ga}_{3} \mathrm{PO}_{7}$ are determined by the frequency-dependent dielectric function $\varepsilon(\omega)=\varepsilon_{1}(\omega)$ $+i \varepsilon_{2}(\omega)$, which is mainly connected to the electronic structures. The imaginary part $\varepsilon_{2}(\omega)$ of the dielectric function $\varepsilon(\omega)$ is calculated from the momentum matrix elements between the occupied and unoccupied electronic states and given by

\footnotetext{
a)Electronic mail: xiaolin@uow.edu.au.
}

$$
\varepsilon_{2}=\frac{2 e^{2} \pi}{\Omega \varepsilon_{0}} \sum_{k, v, c}\left|\left\langle\psi_{k}^{c}|\hat{u} \cdot r| \psi_{k}^{v}\right\rangle\right|^{2} \delta\left(E_{k}^{c}-E_{k}^{v}-E\right),
$$

where $\omega$ is the light frequency, $e$ is the electronic charge, and $\psi_{k}^{c}$ and $\psi_{k}^{v}$ are the conduction band (B) and valence band (VB) wave functions at $k$, respectively. The real part $\varepsilon_{1}(\omega)$ is derived from the imaginary part $\varepsilon_{2}(\omega)$ by the KramersKronig transformation. All the other optical constants, such as the absorption spectrum, refractive index, and reflectivity are derived from $\varepsilon_{1}(\omega)$ and $\varepsilon_{2}(\omega)^{7}$

The structure of $\mathrm{Ga}_{3} \mathrm{PO}_{7}$ comprises $\mathrm{PO}_{4}$ tetrahedral and $\mathrm{GaO}_{5}$ trigonal bipyramids connected by edges to form $\mathrm{Ga}_{3} \mathrm{O}_{10}$ clusters. The structure is isotopic with $\mathrm{Fe}_{3} \mathrm{PO}_{7}$ and $\mathrm{Ga}_{3} \mathrm{PO}_{7}$ is the third known compound in the $\mathrm{Ga}-\mathrm{P}-\mathrm{O}$ system. ${ }^{1,8}$ Figure 1 shows the perspective views of the $\mathrm{Ga}_{3} \mathrm{PO}_{7}$ structure. We used experimentally measured x-ray diffraction data as the initial input for geometry optimization. The lattice parameters was calculated and fitted to third order Birch-Murnaghan equations of states. ${ }^{9,10}$ Table I compares the experimental and calculated data. The results show that the optimized structure expands slightly in comparison to the experimental data, which is natural due to the experimental data is derived from the single crystal grown in a high pressure environment. However, the volume difference is quite small with a value of $2.7 \%$.

The energy band structure is calculated along the highsymmetry directions in the Brillouin zone, as shown in Fig. 2. The total density of states (DOS) and the partial DOS (PDOS) are plotted in Fig. 3.
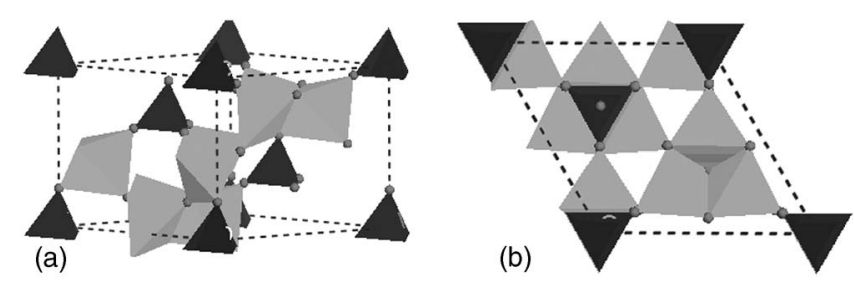

FIG. 1. Crystalline structure of the $\mathrm{Ga}_{3} \mathrm{PO}_{7}$ crystal. (a) Perspective view from original position. (b) Perspective view projected along the $c$ axis with the $a$ axis horizontal and the $b$ axis close to vertical. The $\mathrm{GaO}_{5}$ and $\mathrm{PO}_{4}$ polyhedra are represented in black and light gray. 
TABLE I. Calculated and experimental lattice parameters of $\mathrm{Ga}_{3} \mathrm{PO}_{7}$.

\begin{tabular}{lllll}
\hline \hline & & $a(\AA)$ & $c(\AA)$ & $V\left(\AA^{3}\right)$ \\
\hline $\mathrm{Ga}_{3} \mathrm{PO}_{7}$ & Calc. $^{\mathrm{a}}$ & 7.971 & 6.764 & 372.15 \\
$\mathrm{Ga}_{3} \mathrm{PO}_{7}$ & Expt. $^{\mathrm{b}}$ & 7.885 & 6.727 & 362.20 \\
\hline \hline
\end{tabular}

${ }^{\mathrm{a}}$ This work.

${ }^{\mathrm{b}}$ Reference.

The top of the VB is mainly composed of O $2 p$ states, some P $3 p$ states, and a slight contribution from Ga $4 p$ and $4 s$ states. The bottom of the CB is dominated by $\mathrm{Ga} 4 p$, in addition to a tiny amount of $\mathrm{O} 3 p$ states and $\mathrm{P} 3 p$ states. $\mathrm{Ga}_{3} \mathrm{PO}_{7}$ presents a direct band gap of $3.69 \mathrm{eV}$ at the $\Gamma$ point, which corresponds to an absorption edge of $336 \mathrm{~nm}$. The band gaps predicted by the DFT are usually smaller than the experimental data, which means that the calculated results underestimate the real band gaps implying that the absorption edge should be shorter than $336 \mathrm{~nm}$. In our calculation, the scissor operators on both the electronic structure and the optical properties were not considered. The energy bands below $-20 \mathrm{eV}$ are contributed by P $3 s$ states. The energy bands between -16 and $-20 \mathrm{eV}$ are mainly comprised of $\mathrm{O} 2 \mathrm{~s}$ states and P $3 p$ states. Ga $3 d$ states form the energy bands around $-12 \mathrm{eV}$, which are highly localized, as evidenced by a very sharp peak in the DOS. The states of $\mathrm{O} 2 p, \mathrm{Ga} 4 p$ and $4 s$, and $\mathrm{P} 3 p$ form the energy bands from $-10 \mathrm{eV}$ to the Fermi surface. In the $\mathrm{CB}$, the states are formed by unoccupied $\mathrm{Ga} 4 p$, Ga $4 s, \mathrm{P} 3 s, \mathrm{P} 3 p$, and $\mathrm{O} 2 p$. By analyzing the PDOS, it was found that the $\mathrm{O} 2 p$ states had a strong admixture with P $3 s$ states, as well as Ga $4 p$ and $4 s$ states, which is evidenced by the large number of peaks and the big width of the PDOS of $\mathrm{Ga}$ and $\mathrm{P}$ in the VB region. The small value of the PDOS of the Ga $4 p$ and $4 s$, and P $3 s$ states in this region, in contrast to the large value of $\mathrm{O} 2 p$, indicates the electron transfer to $\mathrm{O} 2 p$ from $\mathrm{P} 3 p$, and Ga $4 p$ and $4 s$. Therefore, $\mathrm{Ga}_{3} \mathrm{PO}_{7}$ has a strong covalent character, involving both $\mathrm{Ga}-\mathrm{O}$ and $\mathrm{P}-\mathrm{O}$ bonds. However, the $\mathrm{P}-\mathrm{O}$ bond is more covalent than the $\mathrm{Ga}-\mathrm{O}$ bond. The bond analysis is in accordance with the character of the structure of $\mathrm{Ga}_{3} \mathrm{PO}_{7}$, with two anion groups of $\mathrm{PO}_{4}$ tetrahedra and $\mathrm{GaO}_{5}$ trigonal bipyramids.

To better understand the bonding behavior of $\mathrm{Ga}_{3} \mathrm{PO}_{7}, \mathrm{~A}$ calculation of the Mulliken charge population was per-

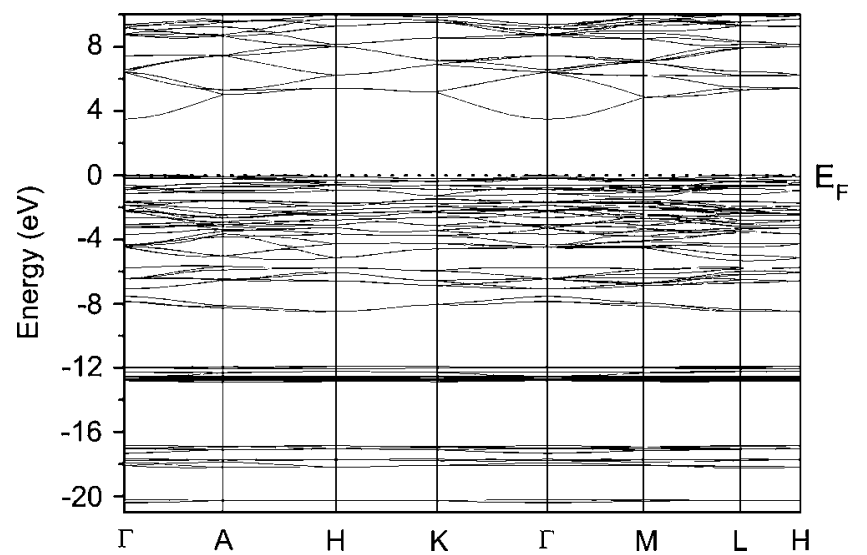

FIG. 2. Energy band structure of the trigonal $\mathrm{Ga}_{3} \mathrm{PO}_{7}$. The high-symmetry $k$ point $\Gamma, \mathrm{A}, \mathrm{H}, \mathrm{K}, \mathrm{M}$, and $\mathrm{L}$ in the figure represent the points $(0,0,1 / 2)$, $(-1 / 3,2 / 3,1 / 2), \quad(-1 / 3,2 / 3,0), \quad(0, \quad 0, \quad 0), \quad(0,1 / 2,1 / 2), \quad$ and $(-1 / 3,2 / 3,1 / 2)$, respectively.

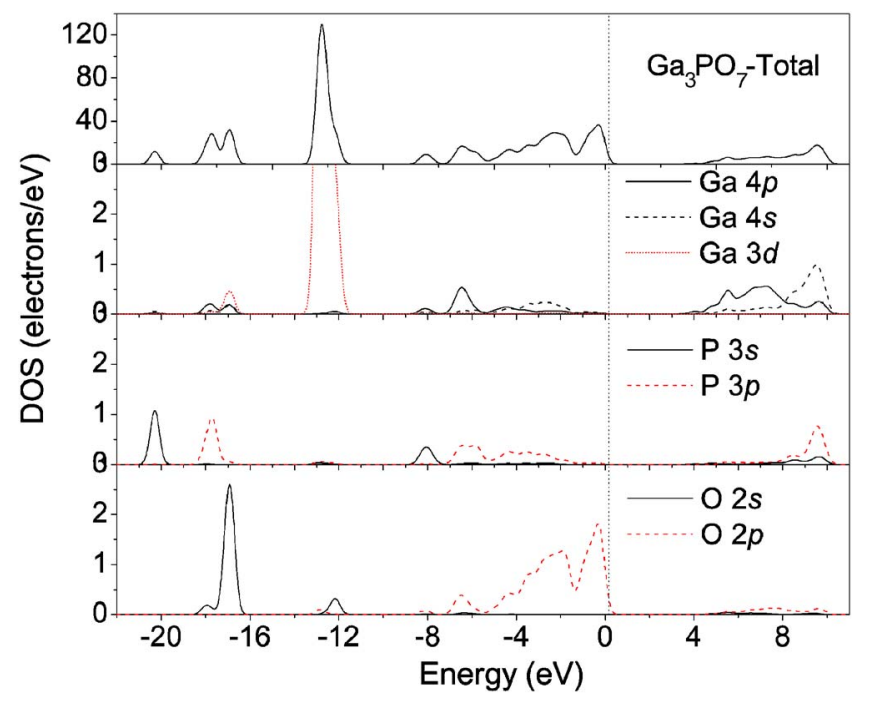

FIG. 3. (Color online) Total DOS and PDOS of trigonal $\mathrm{Ga}_{3} \mathrm{PO}_{7}$.

formed, which is shown in Table II. The charge transfers from $\mathrm{Ga}$ and $\mathrm{P}$ to $\mathrm{O}$ are about $1.52 e$ and $2.27 e$, respectively. The bond order analysis shows that the $\mathrm{P}-\mathrm{O}$ bond has values of 0.59 and 0.71 , depending on the coordination environment, while the $\mathrm{Ga}-\mathrm{O}$ bond has more variable bond order values of $0.55,0.5,0.43$, and 0.14 , depending on the coordination environment in the $\mathrm{Ga}_{3} \mathrm{PO}_{7}$ structure. The closer to 1 of the bond order is the more covalent character the bond has. Therefore, the $\mathrm{P}-\mathrm{O}$ bond shows more covalent character than the $\mathrm{Ga}-\mathrm{O}$ bond.

The calculated dielectric functions of $\mathrm{Ga}_{3} \mathrm{PO}_{7}$ when the incident radiation has linear polarization along the [001] and [100] directions are shown in Fig. 4. Due to the symmetry of the crystal structure, the dielectric function when the incident radiation is polarized along the [100] direction has the same form as the one where the incident polarization is along the [010] direction. The peaks of the imaginary part of the dielectric function are related to the electron excitation. These peaks with relatively strong intensity are located at $6.12 \mathrm{eV}$ (A), $8.2 \mathrm{eV}$ (B), $10.13 \mathrm{eV}$ (C), $15.7 \mathrm{eV}$ (D), $22.1 \mathrm{eV}$ (E), and $26.6 \mathrm{eV}(\mathrm{F})$ for the curve with incident radiation along the $[100] /[010]$ directions. Peaks A, B, and C correspond to the electron transition from the $\mathrm{O} 2 p$ VB to the $\mathrm{Ga} 4 p$ and $4 s$ CB. Peak D corresponds to the transition from the O $2 p$ VB to the $\mathrm{P} 3 p \mathrm{CB}$. Peaks $\mathrm{E}$ and $\mathrm{F}$ are ascribed to the transition of inner electron excitation from $\mathrm{O} 2 s$ and $\mathrm{P} 3 s$ to the CB. The peaks corresponding to electron excitation when the incident light is polarized along the [001] direction are located at similar positions, however, the peak intensities are somewhat different, due to the structure anisotropy of the compound. The calculated static dielectric constants, $\varepsilon_{1}(0)$, are 2.36 and 2.30 , when incident radiation is polarized along the [001] and the [010/100] directions, respectively.

TABLE II. Mulliken charge population of $\mathrm{Ga}_{3} \mathrm{PO}_{7}$

\begin{tabular}{cccccc}
\hline \hline Species & $s$ & $p$ & $d$ & Total $(e)$ & Charge $(e)$ \\
\hline $\mathrm{O}_{1}$ & 1.84 & 5.08 & 0 & 6.92 & -0.92 \\
$\mathrm{O}_{2}$ & 1.82 & 5.22 & 0 & 7.03 & -1.03 \\
$\mathrm{O}_{3}$ & 1.84 & 5.13 & 0 & 6.97 & -0.97 \\
$\mathrm{P}$ & 0.89 & 1.84 & 0 & 2.73 & 2.27 \\
$\mathrm{Ga}$ & 0.54 & 0.95 & 9.99 & 11.48 & 1.52 \\
\hline \hline
\end{tabular}




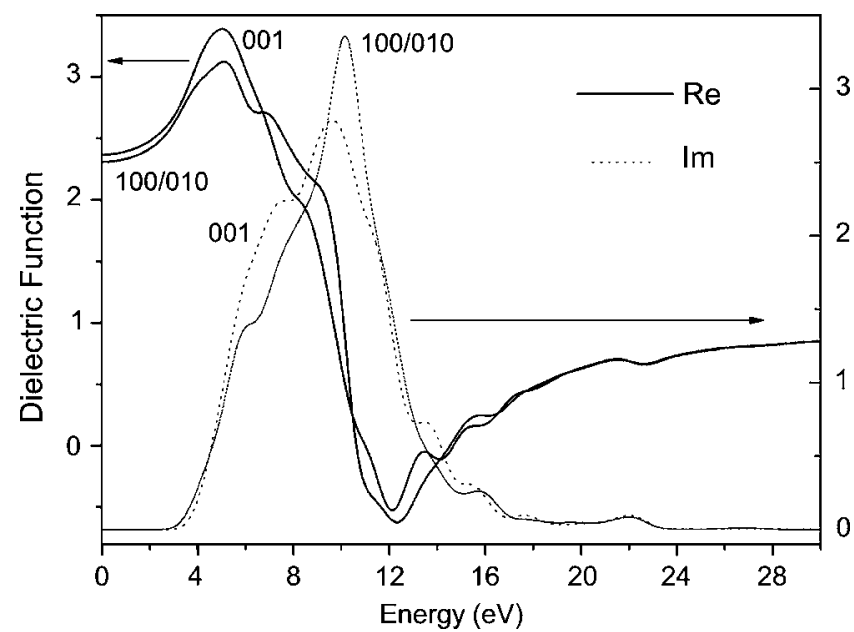

FIG. 4. Anisotropic dielectric function of trigonal $\mathrm{Ga}_{3} \mathrm{PO}_{7}$.

The calculated results on the anisotropic optical absorption spectrum, refractive index, reflectivity, and energy-loss spectrum are shown in Figs. 5(a)-5(d). In our calculation, we use Gaussian smearing of $0.5 \mathrm{eV}$. The optical absorption starts at $3.7 \mathrm{eV}$ for the incident radiation polarized along both the $[100 / 010]$ and the [001] directions, which corresponds to light with a wavelength of $334 \mathrm{~nm}$, which is close to the $350 \mathrm{~nm}$ of the $\mathrm{KTiPO}_{4}$ crystal, a famous nonlinear optical phosphate. ${ }^{11}$ Above $3.7 \mathrm{eV}$, the absorption increases continuously before reaching a maximum. However, the absorption is just $10 \%$ of the peak absorption intensity at $5.10 \mathrm{eV}(242 \mathrm{~nm})$. Simultaneously, this material has a very

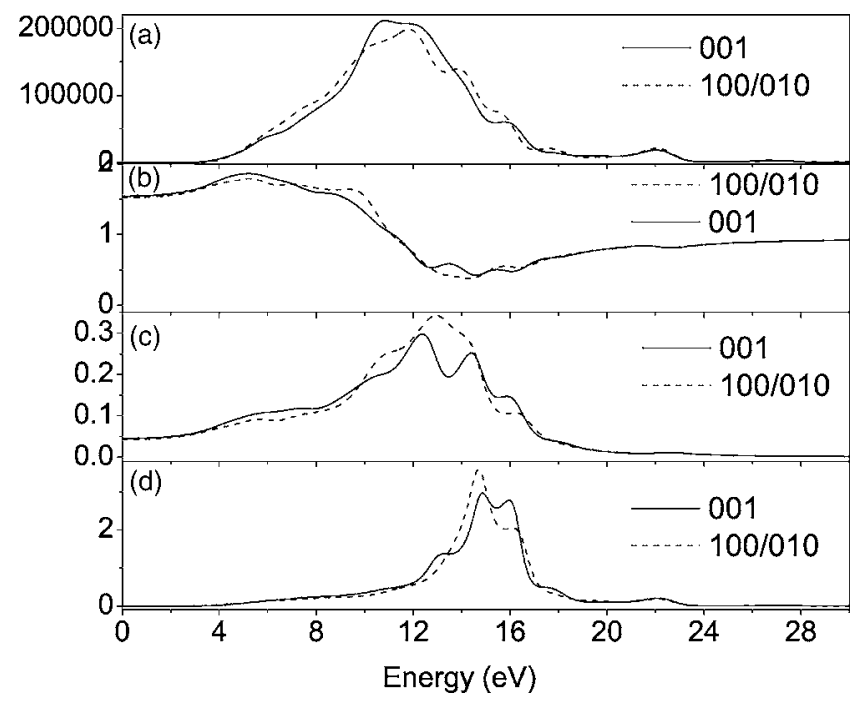

FIG. 5. Anisotropic optical properties of the $\mathrm{Ga}_{3} \mathrm{PO}_{7}$ (a) absorption spectrum, (b) refractive index, (c) reflectivity, and (d) energy-loss spectrum. low reflectivity over a large range of energy for both types of polarized incident radiation $(<0.1$ below $5.17 \mathrm{eV}, 239 \mathrm{~nm})$. These results indicate that $\mathrm{Ga}_{3} \mathrm{PO}_{7}$ is transparent to the light with wavelengths longer than $334 \mathrm{~nm}$. The calculated refractive index is found to be 1.64 at $400 \mathrm{~nm}$ along the two directions, [100/010] and [001], which is very close to the $\sim 1.62$ of $\mathrm{GaPO}_{4}$, a famous piezoelectric phosphate having the same $\mathrm{PO}_{4}$ tetrahedra in its structure as $\mathrm{Ga}_{3} \mathrm{PO}_{7}{ }^{12}$ From the similarity of the values of the absorption edges and the refractive indices of these phosphates, it is obvious that these two properties are decided by $\mathrm{PO}_{4}$ anions group. The energyloss spectrum describes the energy loss of a fast electron traversing the material and is usually large at the plasma energy. Three prominent peaks were found at 13.02, 14.84, and $16.01 \mathrm{eV}$ along the [001] direction, which are consistent with the roots of $\varepsilon_{1}(\omega)$ and correspond to a rapid reduction in the reflectance.

In summary, we calculated the structural parameters, electronic structures, and optical properties of $\mathrm{Ga}_{3} \mathrm{PO}_{7}$ by means of the DFT within GGA. Our structural parameters are in agreement with the experimental data. The electronic structures of $\mathrm{Ga}_{3} \mathrm{PO}_{7}$ show that the top of the $\mathrm{VB}$ and the bottom of the $\mathrm{CB}$ are determined by $\mathrm{O} 2 p$ and $\mathrm{Ga} 4 p$ states, respectively. Although the $\mathrm{P}-\mathrm{O}$ bond shows stronger covalent bonding than the $\mathrm{Ga}-\mathrm{O}$ bond, both bonds are regarded as covalent, which proves that the existence of the two functional anion groups in $\mathrm{Ga}_{3} \mathrm{PO}_{7}$. Anisotropic dielectric and optical properties, including dielectric function, absorption, reflectivity, and energy loss, were calculated and discussed in detail. The results show that $\mathrm{Ga}_{3} \mathrm{PO}_{7}$ has potential applications in optoelectric devices.

This work was supported by the Australian Research Council through Discovery Projects (DP0558753 and DP0665873).

${ }^{1}$ S. Boudin and K.-H. Lu, Acta Crystallogr., Sect. C: Cryst. Struct. Commun. C54, 5 (1998).

${ }^{2}$ G. Xu, J. Li, J. Wang, H. Zhao, X. Cheng, and W. Gao, Appl. Phys. Lett. 92, 101906 (2008).

${ }^{3}$ J. P. Perdew, K. Burke, and M. Ernzerhof, Phys. Rev. Lett. 77, 3865 (1996).

${ }^{4}$ D. Vanderbilt, Phys. Rev. B 41, 7892 (1990).

${ }^{5}$ M. D. Segall, C. J. Pickard, R. Shah, and M. C. Payne, Mol. Phys. 89, 571 (1996).

${ }^{6}$ H. J. Monkhorst and J. D. Pack, Phys. Rev. B 13, 5188 (1976).

${ }^{7}$ S. Saha, T. P. Sinha, and A. Mookerjee, Phys. Rev. B 62, 8828 (2000).

${ }^{8}$ A. Modaressi, A. Courtois, R. Gerardin, B. Malaman, and C. Gleitzer, J. Solid State Chem. 47, 245 (1983).

${ }^{9}$ F. Birch, J. Geophys. Res. 83, 1257 (1978).

${ }^{10}$ F. Birch, Phys. Rev. 71, 809 (1947).

${ }^{11}$ W. Y. Ching and Y. N. Xu, Phys. Rev. B 44, 5332 (1991).

${ }^{12}$ S. Defregger, G. F. Engel, and P. W. Krempl, Phys. Rev. B 43, 6733 (1991). 